\title{
Fusión de sensores de bajo coste para la monitorización de dinámica de vehículos
}

\author{
Pablo Luque Rodríguez \\ Profesor Titular, Universidad de Oviedo, España \\ Daniel Álvarez Mántaras \\ Profesor Titular, Universidad de Oviedo, España \\ Javier García de Jalón \\ Catedrático, INSIA-Universidad Politécnica de Madrid, España \\ José Luis Bueno López \\ Ingeniero Industrial, INSIA-Universidad Politécnica de Madrid, España \\ Jesús Cardenal Carro \\ Profesor Titular, Universidade A Coruña \\ Álvaro Deibe Díaz \\ Profesor Titular, Universidade A Coruña
}

\section{RESUMEN}

La caracterización dinámica de vehículos, permitiendo obtener la posición y orientación espacial de un vehículo en cada instante de tiempo, es fundamental no sólo evaluar cómo se comporta dinámicamente el vehículo y de esa forma hacer los ajustes necesarios en sus componentes para conseguir unas prestaciones determinadas, sino que se trata de, junto con otros sistemas, ser capaces de guiar el vehículo y realizar incluso una "conducción inteligente”. La aplicación de sistemas de caracterización dinámica mediante el uso de sensores de bajo coste en vehículos terrestres es relativamente reciente y está en auge en los últimos años por el gran desarrollo de estos sensores.

El presente trabajo presenta los desarrollos de una línea de investigación seguida por los autores que tiene como finalidad investigar las distintas posibilidades que ofrece la fusión de sensores para caracterizar dinámicamente un sistema móvil. El objetivo fundamental es conseguir desarrollar un sistema que pueda ser implementado sobre un vehículo y permita estimar la posición y orientación del mismo, proporcionando información del valor de sus estados en cada instante. Con ello, se pretende ser capaz de evaluar el comportamiento dinámico del automóvil.

Se estudian diferentes técnicas para lograr la estimación de los estados de un sistema y se analizarán los distintos tipos de sensores que se pueden emplear para la toma de medidas y el proceso de fusión de los mismos. Todo ello, con la intención de desarrollar una herramienta que permita determinar, de la forma más precisa posible y con unos costes reducidos, aquellas variables que definen la dinámica de los vehículos: balanceo, cabeceo, guiñada, velocidades angulares, aceleraciones, etc. 


\section{INTRODUCCIÓN}

La reducción del peso de los vehículos es uno de los caminos para la reducción del consumo de combustible y de emisiones contaminantes. En el caso de los vehículos industriales una reducción del peso en vacío supone además un aumento de la capacidad de carga útil. Por tanto, en este tipo de vehículos tiene una repercusión superior a la que tiene en otro tipo de vehículos.

En los vehículos de grandes dimensiones (semirremolques, por ejemplo) y los autobuses, la estructura supone una parte muy representativa del peso en vacío del vehículo. Por tanto, su aligeramiento repercute significativamente en el peso final. Este aligeramiento debe hacerse manteniendo las condiciones de seguridad y garantizando una vida útil adecuada. Pero, en cualquier caso hay que indicar que el aligeramiento puede afectar a la Resistencia al envejecimiento del bastidor y del conjunto del vehículo. El aligeramiento de la estructura afecta a la vida a fatiga, por tanto, para garantizar que éste no afecta al envejecimiento es necesario llevar a cabo un diseño basado en un estudio preciso de la vida a fatiga. Para ello, es necesario conocer las cargas a las que va a estar sometida la estructura durante su funcionamiento y, con ello, realizar el diseño optimizado en base a la utilización de técnicas de simulación por elementos finitos combinados con técnicas de postprocesado numérico de las tensiones obtenidas en los estados de carga simulados.

Para la determinación de las cargas en servicio se ha planteado una metodología basada en la simulación de modelos virtuales a partir de la entrada de datos reales de circulación obtenidos mediante una instrumentación de bajo coste. La combinación de adquisición de datos reales más modelo virtual para la optimización de los diseños es un método que ha sido aplicado con éxito a otro tipo de vehículos. La ventaja de combinar ambos sistemas es que se reduce la complejidad del sistema de adquisición de datos y el número de sensores necesarios. En los vehículos objeto de estudio las cargas necesarias para un cálculo de la vida a fatiga son debidas a las acciones de los sistemas de tracción y freno, efectos de la suspensión, la carga, etc. Esto implicaría la necesidad de una instrumentación muy compleja, por tanto, no sería aplicable a flotas de vehículos.

En el presente trabajo se propone es un sistema de monitorización dinámica aplicable a flotas vehículos industriales o autobuses, desarrollando para ello una instrumentación no intrusiva, robusta y de bajo coste. El sistema propuesto se basa en una combinación de una unidad de medida inercial y uno o varios receptores para el posicionamiento global con sensores de bajo coste.

\section{SISTEMAS DE NAVEGACIÓN Y SENSORES}

Para la medición de la velocidad de un punto de referencia (centro de gravedad, King pin en el caso de un semirrolque,...) la instrumentación más simple pasa por la utilización de un receptor de posición global, cuya salida es velocidad absoluta y ángulo de ruta. La velocidad leída directamente del receptor presenta dos problemas, un nivel de ruido 
elevado y la baja precisión o ausencia de lectura por una mala señal de recepción. Estos problemas, dado el entorno en el que circulan los vehículos, se producen con frecuencia. El ruido en la señal del sistema de posicionamiento global supone variaciones importantes de la velocidad, lo cual implicaría entradas al modelo virtual que suponen variaciones importantes de aceleración. Estas variaciones, durante la simulación, dan lugar a cargas elevadas muy superiores a las cargas reales de circulación. El análisis de fatiga, basado en cargas obtenidas de esta manera, no es fiable. Un filtrado de esta señal no soluciona este problema.

Por otro lado, la medición de la velocidad de un punto característico, como el centro de gravedad o el King pin con un receptor de posición global (una antena GPS, por ejemplo) no puede aplicarse a cualquier tipo de vehículo, dado que éste punto suele estar oculto bajo la caja o la carga, siendo aplicable únicamente a semirremolques plataforma o portacontenedores. Es por ello que deben analizarse las posibles soluciones en cuanto a sensorización y sistemas de navegación disponibles en la actualidad.

La navegación es una técnica que consiste en la determinación de la posición y la velocidad de un móvil con respecto a un sistema de referencia conocido, determinando en algunos casos su orientación también. Un sistema de navegación puede ser autónomo o depender de agentes externos o, en algunos casos, puede ser resultado de la combinación de ambos. Existen fundamentalmente dos métodos de operación:

- "Dead Reckoning” (DR): Son sistemas autónomos y emplean la información de velocidad, posición y rumbo iniciales para estimar el valor que tendrán los mismos en determinado instante de tiempo. En este grupo se pueden incluir las siguientes técnicas:

Odometría: técnica que emplea sensores instalados en el sistema móvil para obtener una aproximación de la posición real en la que se encuentra dicho sistema en un determinado instante de tiempo con respecto a un sistema de referencia inicial. Los datos se obtienen, por ejemplo, a partir de sensores que miden la rotación de una rueda. Las ventajas de este método están en su precisión, bajo coste y la posibilidad de usar una frecuencia de muestreo elevada. El inconveniente es que, por ejemplo en el caso indicado, no tiene en cuenta el deslizamiento de la rueda. Además, teniendo en cuenta que lo que hace es integrar en el tiempo la información que tiene del movimiento del vehículo (la distancia recorrida), si aparecen errores estos se incrementen cada vez que se produzca una nueva integral. En concreto, los errores en la orientación provocan granes errores a la hora de determinar la posición, lo cual empeora mucho la precisión de la solución final que se consigue mediante esta técnica.

Navegación Inercial (Inertial Navigation System o INS) La navegación inercial emplea sensores de tipo inercial (Inertial Measurement Unit o IMU), giróscopos y acelerómetros, que miden la rotación y las fuerzas que 
actúan sobre el móvil para determinar su aceleración. Comenzando en una posición y orientación conocida, las medidas son integradas una vez por el giróscopo y dos por el acelerómetro para proporcionar la orientación y posición del móvil respectivamente. Si hay un error tiende a acumularse en el tiempo, lo cual quiere decir que este tipo de sistema está afectado por la deriva del error del tiempo.

- "Position fixing": Son métodos de toma de medidas directas de la posición del móvil. Se basan en la información captada por fuentes externas ubicadas en posiciones conocidas. En este grupo se pueden incluir:

Brújulas electrónicas: Aunque no proporcionan la posición por ellas mismas, una brújula electrónica es un dispositivo que emplea un magnetómetro para proporcionar medidas del rumbo del vehículo en relación con el norte magnético de la tierra. Esta técnica necesita conocer el ángulo de declinación magnética, que es función de la posición. Presenta el inconveniente de que los campos magnéticos se distorsionan fácilmente por la proximidad de estructuras metálicas u otras líneas de campo durante la trayectoria del vehículo, lo cual origina grades e impredecibles errores en la estimación del rumbo. Esto hace que el uso de estos sistemas para aplicaciones de navegación en vehículos terrestres no sea recomendable.

Balizas de seguimiento: Este método se emplea sobre todo en aplicaciones en las que el movimiento de la plataforma se realiza en un entorno conocido, donde capaz de proporcionar medidas muy precisas. Aparte de diversos problemas de aplicación, no resulta de gran utilidad en sensorización de vehículos de carretera en condiciones de tráfico abierto.

Navegación mediante puntos de referencia: Es similar al sistema utilizado por las balizas. Para poder aplicarlo se necesita que el móvil se mueva en un entorno bien conocido. Los puntos de referencia o marcas son formas geométricas que pueden ser detectadas por sensores en el vehículo. Al igual que en el sistema de balizas, requiere mucho mantenimiento y se usa solo en aplicaciones muy concretas.

Posicionamiento basado en mapas: Se emplea cuando la plataforma móvil se mueve en un entorno que es conocida $\mathrm{y}$ ha sido cartografiado previamente. El vehículo posee sensores que captan datos del entorno en el cual se está moviendo y los compara luego con un mapa que tiene almacenado para determinar su ubicación. Cámaras y dispositivos de luz láser son el tipo de sensores empleados en este sistema. Este método solo se puede utilizar en entornos que están bien estructurados o definidos, lo que significa que es útil sobre todo en interiores y algunos exteriores bien conocidos. Este sistema de mapas se usa combinado con receptores GPS para determinar el recorrido de un vehículo por una ciudad.

Sistemas de navegación global mediante satélites: (Global Navigation 
Satellite Systems o GNSS). Estos sistemas se basan en constelaciones de satélites que emiten señales de radio codificadas. Mediante un proceso de trilateración y utilizando la información de posición de los satélites, el tiempo que tardan en recibir las señales, la velocidad de las señales y las distancias a los satélites; calcula la posición en que se encuentran en términos de latitud, longitud y altura geodésicas. Estos sistemas proporcionan una solución muy buena para a navegación en exteriores, pero en interiores o con presencia de obstáculos no son una solución tan buena, ya que se pierde la señal de los satélites. Existen en la actualidad diversos sistemas GNSS operativos o en curso de serlo: el NAVSTAR-GPS desarrollado y operado por la fuerza aérea estadounidense y operativo desde 1992, el sistema GLONASS desarrollado y operado por el ministerio de defensa ruso, que actualmente se encuentra en proceso de reparación, y el sistema GALILEO, único de origen civil, desarrollado en Europa, que en un principio iba comenzar a funcionar en 2014.

Existen además sistemas combinados, en los que se utilizan medidas relativas (por ejemplo sensores de movimiento) y medidas absolutas. Una práctica habitual es emplear sistemas de los dos grupos de forma combinada para determinar la posición del móvil de la forma más precisa posible. De todas las técnicas anteriormente descritas, las más utilizadas con los sensores inerciales y sistemas de posicionamiento con satélites.

Los avances de la tecnología y la electrónica, y por lo tanto la reducción del tamaño de los componentes de los circuitos integrados ha facilitado la implementación de este tipo de sistemas. Esto ha permitido que equipos que en un primer momento se utilizaban con exclusividad para aplicaciones espaciales y militares, puedan ser utilizados con otros fines no tan sofisticados que en los que se disponga de un presupuesto tan alto.

\section{FUSIÓN DE SENSORES}

\subsection{Introducción}

La fusión de los datos procedentes de distintos sensores es un método que consiste en combinar información procedente de diferentes fuentes con el objetivo de proporcionar una descripción completa y detallada del proceso de interés. Esta práctica posee ventajas significativas sobre una fuente única de información, ya que permite tener más datos y conseguir resultados más precisos. Tiene aplicaciones en varias disciplinas, no se emplea solamente en la caracterización dinámica de vehículos, sino que también se utilizan en sistemas militares y espaciales, en la vigilancia y en tareas de supervisión civil, en control de procesos, robótica y medicina. Es un proceso particularmente importante si se desea desarrollar cualquier tipo de sistema autónomo. Un punto importante en la fusión de datos procedentes de distintos sensores es el desarrollo de modelos apropiados de la incertidumbre asociada con los estados y el modelo de observación y de los algoritmos que 
permitan fusionar los daros procedentes de dispositivos diferentes.

En el caso de la caracterización dinámica de un vehículo, la fusión de sensores consiste en utilizar de forma conjunta sensores inerciales y sensores de posicionamiento respecto a una referencia fija (GNSS), para obtener información de la posición, velocidad y orientación de la forma más precisa posible, al ser capaz de eliminar los errores presentes en las medidas aprovechando las ventajas de cada uno de los dispositivos. Las señales de los GNSS se utilizan para corregir los errores de deriva que tienen los giróscopos, y los sensores inerciales se utilizaban como fuente de información cuando la señal del satélite llega con demasiado ruido o no llega debido a los obstáculos.

En un primer momento los sensores inerciales tenían un precio elevadísimo y su empleo solo era asumible en aplicaciones que requiriesen una precisión muy elevada. El desarrollo de los MEMS (Micro-Electro-Mechanical-Systems) originó una reducción importante en los costes de los giróscopos y acelerómetros haciendo así posible su utilización junto con los GNSS (GPS, por ejemplo) en sistemas de navegación de bajo coste que pueden ser implementados en vehículos terrestres.

\subsection{Integración GNSS/INS}

Debido a los diferentes principios de operación que utilizan cada uno de los dispositivos, los GNSS y los sensores inerciales suelen utilizarse de forma conjunta para determinar tanto posición, orientación como la velocidad de un vehículo. Esto es debido a que la precisión de los resultados será mucho mayor que la que se consigue al implementar cada dispositivo por separado. Los datos de estos sistemas pueden ser combinados debido a las diferencias entre las características de ambos, una de las formas más comunes de realizar este proceso es utilizando un filtro Kalman, pudiendo obtener de esta forma los siguientes objetivos:

- Mayor precisión a la hora de determinar la posición y la velocidad de un móvil.

- Información precisa de la orientación.

- Una cantidad elevada de datos utilizando para ello una frecuencia de muestreo alta.

- Una solución eficaz a algunos de los errores que se producen en la señal de los receptores GNSS, como puede ser el bloqueo de la señal.

Existen diferentes arquitecturas para integrar GNS y INSS pudiendo así conseguir las mejores situaciones posibles del sistema en función del tipo de uso y el grado de simplicidad o robustez necesario en el sistema o la aplicación del mismo. Las tres estructuras básicas utilizadas en la integración son:

- Integración INS/GNSS descentralizada o suave: Dentro de este tipo de estructura el GNSS y el INS funcionan de forma independiente proporcionando soluciones independientes para la posición, orientación y velocidad del móvil. Para conseguir el mejor resultado se fusionan ambos datos con el optimizador adecuado, con ello se obtiene una tercera solución 
en la que la precisión sea mucho más elevada.

- Integración INS/GNSS centralizada o fuerte: En este tipo de arquitectura el GNSS y el INS trabajan como sensores básicos. Las salidas sin procesar (las pseudodistancias en el caso de los GNSS, y las aceleraciones y los ángulos de giro en los INS) son fusionadas por un optimizador estimador para conseguir una solución sinérgica.

- Integración INS/GNSS con realimentación: En esta estructura se produce una elevada fusión entre el GNSS y el INS, ya que emplea un bucle de realimentación. La principal ventaja de esta estructura es que la dinámica del vehículo sobre el que se monta es estimada y compensada en la cadena del GNSS usando la información del efecto Doppler.

En el presente trabajo, debido al tipo de sensores utilizado (de bajo coste) y a la aplicación del sistema (implementable en una flota de vehículos industriales), se optará por una integración descentralizada o suave (Loosely coupled INS/GNSS integration).

\subsection{El Filtro de Kalman Extendido (Extended Kalman Filter o EKF)}

El filtro de Kalman es un algoritmo desarrollado por Rudolf E. Kalman en 1960 que sirve para identificar el estado oculto (no medible) de un sistema dinámico lineal y funciona adecuadamente además cuando el sistema está sometido a ruido blanco aditivo. En este tipo de formulación la ganancia $K$ de realimentación del error se escoger de forma óptima cuando se conocen las varianzas de los ruidos que afectan al sistema. Debido a que el filtro de Kalman es un algoritmo recursivo, este puede correr en tiempo real usando únicamente las mediciones de entrada actuales, es estado calculado previamente y su matriz de incertidumbre, y no requiere alguna otra información pasada adicional. Desde el punto de vista de los problemas que involucran control y estimación, el filtro de Kalman ha sido considerado el gran logro en la teoría de estimación. El principal uso del filtro ha sido en los sistemas de control modernos, en el seguimiento y navegación sobre todo tipo de vehículos, y en el diseño predictivo de estimación de los mismos.

Dado un sistema físico, mediante la aplicación de leyes de la física y el ensayo se busca encontrar la relación entre las variables de interés, es decir, definir un modelo matemático que represente de forma adecuada alguno de los aspectos del comportamiento del sistema a estudiar. Con ese modelo es posible predecir cuál será su próximo estado. Hay varias razones por las cuales un sistema se este tipo no proporcionan una información que en muchos casos no es del todo fiable:

$\checkmark$ Los modelos matemáticos con los que se aproximan los procesos no son del todo exactos. En su creación solo se tienen en cuenta los aspectos más relevantes del mismo, concretamente aquellos que su creador consideró de mayor importancia.

$\checkmark$ Las entradas al sistema y los datos que en la medida del mismo se utilizan suelen estar afectadas por perturbaciones. Estas perturbaciones por norma general no pueden ser controladas con lo cual no se tendrá un modelo matemático con el cual 
aproximar su comportamiento.

$\checkmark$ Todo aparato de medida tiene una precisión limitada y pueden incluir entre sus medidas ruidos y distorsiones debido simplemente a la manera que tienen de captar las mediciones entre otras cosas.

Si se tiene esto en cuenta lo anteriormente descrito será imposible conocer perfectamente el comportamiento del sistema con lo cual será una tarea infructuosa intentar controlarlo utilizando para ello sistemas determinísticos. Es necesario por lo tanto buscar modelos estocásticos de esta forma será posible tener en cuenta las incertidumbres que los modelos deterministas no son capaces de calcular. Es decir con ellos es posible tener cierto control sobre los sistemas sometidos a perturbaciones.

Por lo tanto en este estudio se utilizará un filtro de Kalman, dado que es un conjunto de ecuaciones matemáticas que proveen una solución recursiva eficiente del método de mínimos cuadrados. Esta solución permite calcular un estimador lineal, insesgado y óptimo del estado de un proceso en cada momento del tiempo con base en la información disponible en el momento $t-1$, y actualizar, con la información adicional disponible en el momento $t$, dichas estimaciones. Este filtro es el principal algoritmo para estimar sistemas dinámicos especificados en la forma de estado-espacio. La representación estado-espacio es esencialmente una notación conveniente a la estimación de modelos estocásticos donde se asumen errores en la medición del sistema, lo que permite abordar el manejo de un amplio rango de modelos de series de tiempo.

El filtro es un procedimiento matemático que opera por medio de un mecanismo de predicción y corrección. En esencia este algoritmo pronostica el nuevo estad a partir de su estimación previa añadiendo un término de corrección proporcional al error de predicción, de tal forma que este último es minimizado estadísticamente. Dentro de la notación estadoespacio, la derivación del filtro de Kalman descansa en el supuesto de normalidad del vector de estado inicial y de las perturbaciones del sistema. De tal forma que es posible calcular la función de verosimilitud sobre el error de predicción del modelo son generados por el filtro. Estos son utilizados para evaluar recursivamente la sunción de verosimilitud hasta maximizarla.

Sin embargo, en el caso de que el proceso que se pretenda estimar o la relación existente entre las medidas y el proceso no sean lineales, el filtro Kalman no es aplicable, por lo que en estos casos se aplica el llamado filtro Kalman extendido o en inglés Extended Kalman Filter (EKF). En estos casos se asume que el proceso objeto de estudio tiene el vector de estado $x \in \mathcal{R}^{n}$, controlado por una ecuación diferencial no lineal que presenta una forma como la que se puede observar en la ecuación siguiente:

$$
x_{k}=f\left(x_{k-1}, u_{k-1}, w_{k-1}\right)
$$


Siendo $\boldsymbol{z} \in \mathcal{R}^{m}$ el vector de medidas del proceso, el cual puede expresarse como:

$$
z_{k}=h\left(x_{k}, v_{k}\right)
$$

En las ecuaciones anteriores las variables aleatorias $w_{k}$ y $v_{k}$ representan el ruido del proceso y de las medidas respectivamente. En este caso, la función no lineal $f$ en la ecuación (1) relaciona el estado anterior $k-1$ con el estado actual $k$. En esa misma ecuación se puede encontrar el término $u_{k}$, o función de control. Analizando la función relativa a las medidas (2), la función $h$, no lineal, relaciona el vector de estados $x_{k}$ con el de las medidas $z_{k}$. En la práctica, los valores del ruido $w_{k}$ y $v_{k}$ no son conocidos en cada paso del filtro, sin embargo se puede aproximar el vector de estados y de medias, sin tener en cuenta estos ruidos, mediante las expresiones siguientes:

$$
\begin{gathered}
\tilde{x}_{k}=f\left(\hat{x}_{k-1}, \hat{u}_{k-1}, 0\right) \\
\tilde{z}_{k}=h\left(\tilde{x}_{k}, 0\right)
\end{gathered}
$$

Donde $\hat{x}_{k}$ es la estimación a posteriori de los estados. Para estimar un proceso utilizando ecuaciones diferenciales no lineales se necesitan expresiones que permiten linealizar las estimaciones realizadas. Estas expresiones son las que se pueden ver en las ecuaciones (5) y (6).

$$
\begin{gathered}
x_{k} \approx \tilde{x}_{k}+F\left(x_{k-1}-\hat{x}_{k-1}\right)+W w_{k-1} \\
z_{k} \approx \tilde{z}_{k}+H\left(x_{k-1}-\hat{x}_{k-1}\right)+V v_{k-1}
\end{gathered}
$$

Donde:

$x_{k}$ y $z_{k}-$. Son el vector de estados y el de medias en el instante actual respectivamente.

$\tilde{x}_{k}$ y $\tilde{z}_{k}-$. Son las aproximaciones del vector de estados y el de medidas, correspondientes a las ecuaciones (3) y (4).

$\hat{x}_{k}-$. Es la estimación a posteriori del estado para el paso actual $k$.

$w_{k}$ y $v_{k}-$. Son los valores del ruido del proceso y de las medidas.

$F$-. Es la matriz Jacobiana de derivadas parciales de la función $f$ respecto de $x$, que toma la forma:

$$
F_{[i, j]}=\frac{\partial f(i)}{\partial x(j)}\left(\hat{x}_{k-1}, \hat{u}_{k-1}, 0\right)
$$

$W$-. Es la matriz Jacobiana de derivadas parciales de la función $f$ respecto a $w$.

$$
W_{[i, j]}=\frac{\partial f(i)}{\partial w(j)}\left(\hat{x}_{k-1}, \hat{u}_{k-1}, 0\right)
$$

$H-$. Es la matriz Jacobiana de derivadas parciales de la función $h$ respecto a $x$.

$$
H_{[i, j]}=\frac{\partial h(i)}{\partial x(j)}\left(\tilde{x}_{k}, 0\right)
$$


$V$-. Es la matriz Jacobiana de derivadas parciales de la función $h$ respecto a $v$.

$$
V_{[i, j]}=\frac{\partial h(i)}{\partial v(j)}\left(\tilde{x}_{k}, 0\right)
$$

El algoritmo utilizado se presenta en la figura 1. Dentro del filtro extendido es importante tener en cuenta que el Jacobiano, $H_{k}$, contenido en la ecuación de la ganancia de Kalman, sirve para "amplificar" la componente más importante de la medida. Es decir, si no existe una relación directa entre el vector de estados $z_{k}$ y el de estados $x_{k}$ a través del vector $h$, con la matriz $H_{k}$ la ganancia resulta afectada de tal manera que sólo amplifica la parte del residuo $z_{k}-h\left(\hat{x}_{k}^{*}, 0\right)$ que afecta al estado. Esto implica que si entre todas las medidas y el estado no existiera una relación directa vía $h$, el filtro divergiría rápidamente. En dicho caso el proceso sería no observable.

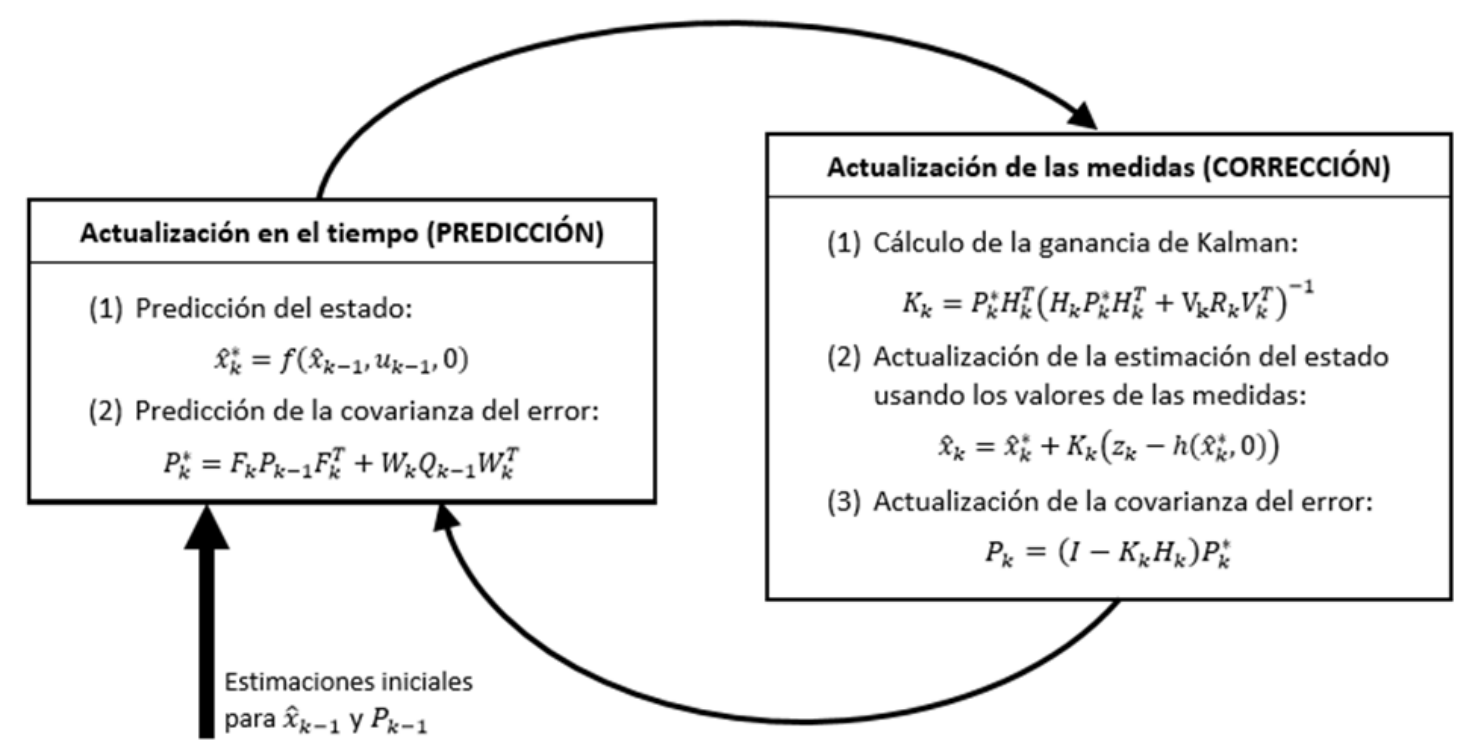

Fig. 1 - Algoritmo del Filtro Kalman extendido

\section{APLICACIÓN DEL SISTEMA DESARROLLADO}

En el presente trabajo se presenta el desarrollo de un sistema para conocer el comportamiento dinámico de un vehículo terrestre a través de la implantación en el mismo de un sistema formado por dispositivos de navegación de bajo coste. Este sistema constará, en este caso, de tres antenas o receptores GNSS y una unidad de medida inercial o IMU. En este caso se ha aplicado a un semirremolque para el transporte de mercancías por carretera, tal y como se muestra en la figura 2. De forma adicional se instala una unidad de medida inercial de precisión con objeto de poder validar los resultados obtenidos. Los receptores GNSS de bajo coste serán de la marca Racelogic, del modelo VBOX Sport, que son dispositivos portátiles que pueden ser colocados con cualquier tipo de vehículo y pueden ser conectados directamente a un teléfono móvil en el cual pueden verse los resultados directamente. Es un receptor GPS diseñado principalmente para uso en vehículos de carretera. Los datos que se pueden obtener con el receptor GPS son: posición, 
rumbo, velocidad y aceleración.

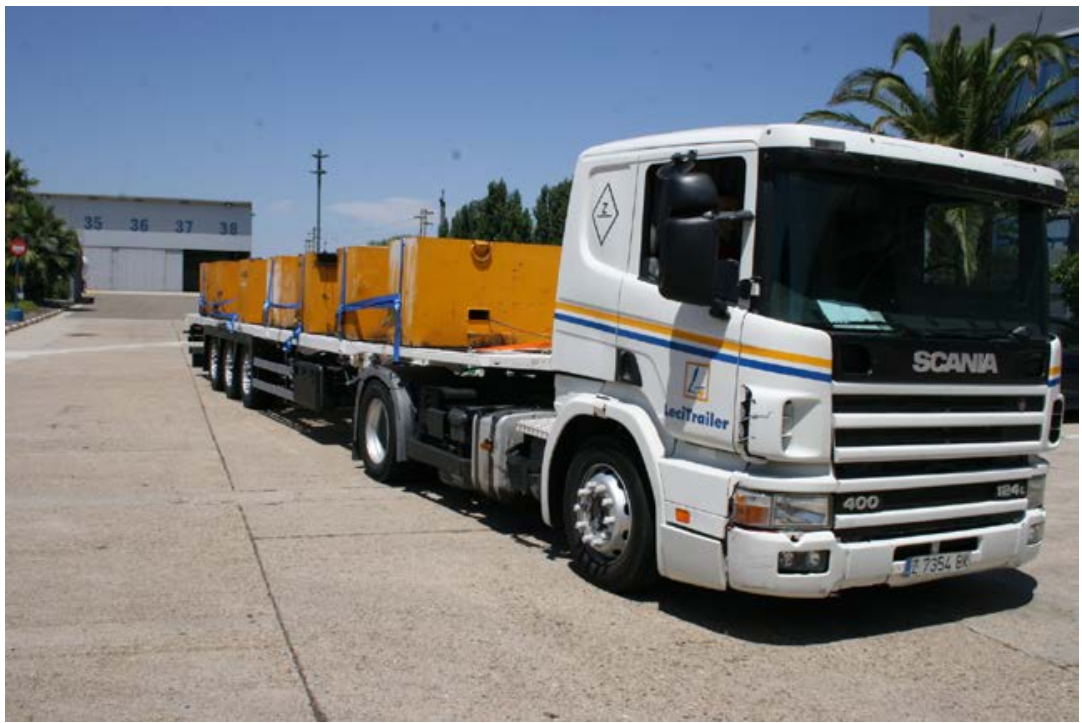

Fig. 2 - Vehículo instrumentado con el sistema desarrollado
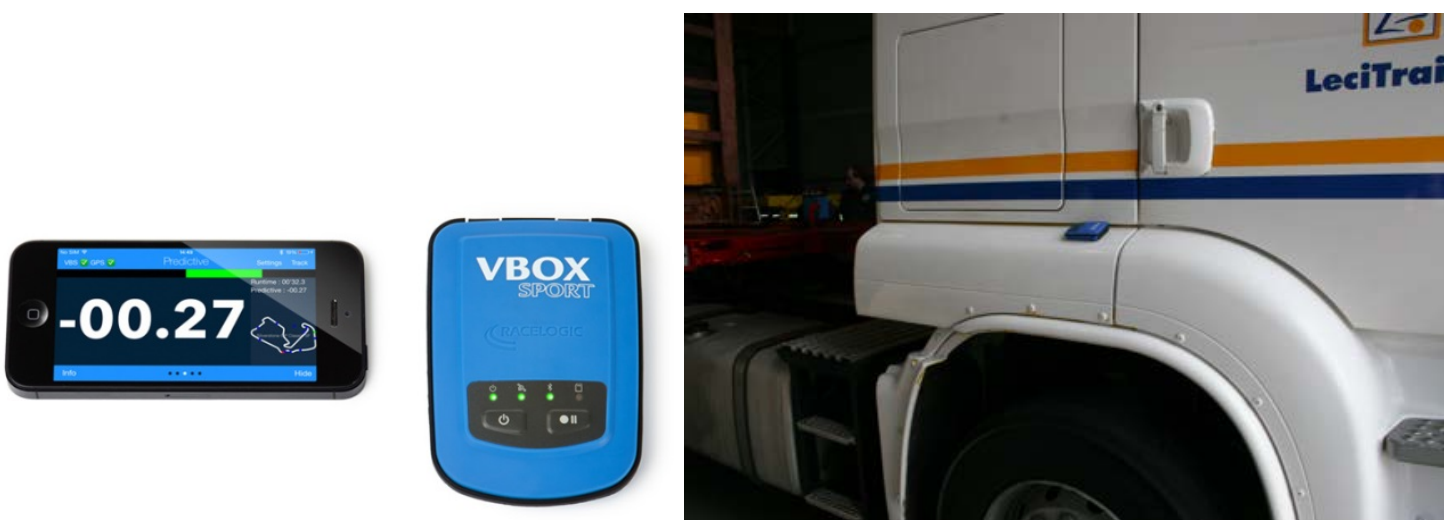

Fig. 3 Receptor GNSS de bajo coste empleado (VBOX Sport)

La IMU empleada conjuntamente constará de tres acelerómetros y tres giroscopios conjuntamente, será el modelo UM7 de la empresa CH Robotics. El UM7 es una IMU de tercera generación y la rúbrica del Sistema de Referencia (AHRS) que aprovecha la tecnología de última generación MEMS para mejorar el rendimiento y reducir los costes. Combina acelerómetro triaxial, giroscopio, magnetómetro y datos utilizando un sofisticado filtro de Kalman extendido para producir sus medidas y estimaciones de partida. Con objeto de valorar los resultados obtenidos se utilizará una IMU de gran precisión del fabricante Genesys, concretamente el modelo ADMA G Entry Level.

Con el objetivo de analizar diferentes configuraciones de los sensores, se han propuesto diversas arquitecturas del sistema de captación, constando cada una de las alternativas constará de diferentes dispositivos de medida colocados en diferentes partes del vehículo, tal y como se especifica a continuación:

- Caso 0: Únicamente constará de una unidad de medida inercial o IMU instalada en 
el pivote de acoplamiento (King-Pin) del vehículo.

- Caso 1: Constará de un receptor GPS colocado en el pivote o “King Pin” (KP), y una unidad de medida inercial o IMU instalada en el mismo punto.

- Caso 2: El sistema estará compuesto un receptor GPS en la esquina trasera derecha del semirremolque junto con una unidad de medida inercial en el pivote.

- Caso 2b: El sistema será igual que el anterior pero en este caso la posición del GPS será la esquina trasera izquierda.

- Caso 3: Se colocarán de dos receptores GPS, uno en la esquina trasera derecha del semirremolque y otro en la izquierda. También incluirá una unidad de medida inercial en el pivote.

- Caso 4: Constará con además de dos receptores GPS, uno en la esquina trasera derecha del semirremolque y otro en la izquierda, con otro en el King Pin. Junto con el tercer GPS estará colocada una IMU.

Para validar tanto el diseño del filtro realizada como su implementación matemática en MATLAB, se realizará una verificación con modelos virtuales utilizando para ello herramientas de simulación multicuerpo con el programa ADAMS, en el cual se representará un modelo virtual del vehículo con los sensores colocados en los puntos especificados.

\section{RESULTADOS Y CONCLUSIONES}

Tras la realización de un amplio programa experimental con el vehículo y los sensores indicados, se procede a la extracción de resultados y el análisis de los mismos. Se dispone por tanto de una gran cantidad de información sobre variables cinemáticas (posiciones y trayectorias, velocidades y aceleraciones) de los diversos puntos de medida, en función de la arquitectura del sistema. Como métricos utilizados para la evaluación coste/beneficio de cada una de las alternativas propuestas, se analiza el error en la estimación de las velocidades longitudinal y transversal.

Como ejemplo de los resultados obtenidos por la implementación del sistema y su aplicación en un caso real de un vehículo circulando en una situación de tráfico abierto, en la figura 4 se muestran los resultados obtenidos en la estimación de la velocidad longitudinal. Se puede observar una disminución progresiva del valor de los mismos del Caso 1 al Caso 4, lo que indica que las previsiones realizadas se cumplen, a mayor cantidad de dispositivos implicados mayor es la precisión del resultado generado, tal y como era de esperar, pero ello implica un mayor coste de adquisición e implantación. Los resultados obtenidos son la base para la realización de un análisis coste/beneficio en el caso de la implantación del sistema desarrollado sobre una flota real objeto de estudio.

A modo de conclusión final se puede indicar que se ha desarrollado una metodología que permite la instrumentación de vehículos de carretera basada en la fusión sensores de bajo coste y es aplicable a la monitorización de una flota de vehículos de grandes dimensiones, 
tanto para el transporte de mercancías como de pasajeros por carretera. El sistema ha sido validado mediante la realización de un programa experimental con modelos virtuales y posteriormente ha sido aplicado a un vehículo real circulando en condiciones reales de tráfico abierto. El sistema desarrollado es aplicable por tanto para la monitorización de este tipo de vehículos, para la extracción de información (cargas, desplazamientos,...) necesarias para la optimización estructural y los estudios de vida a fatiga.

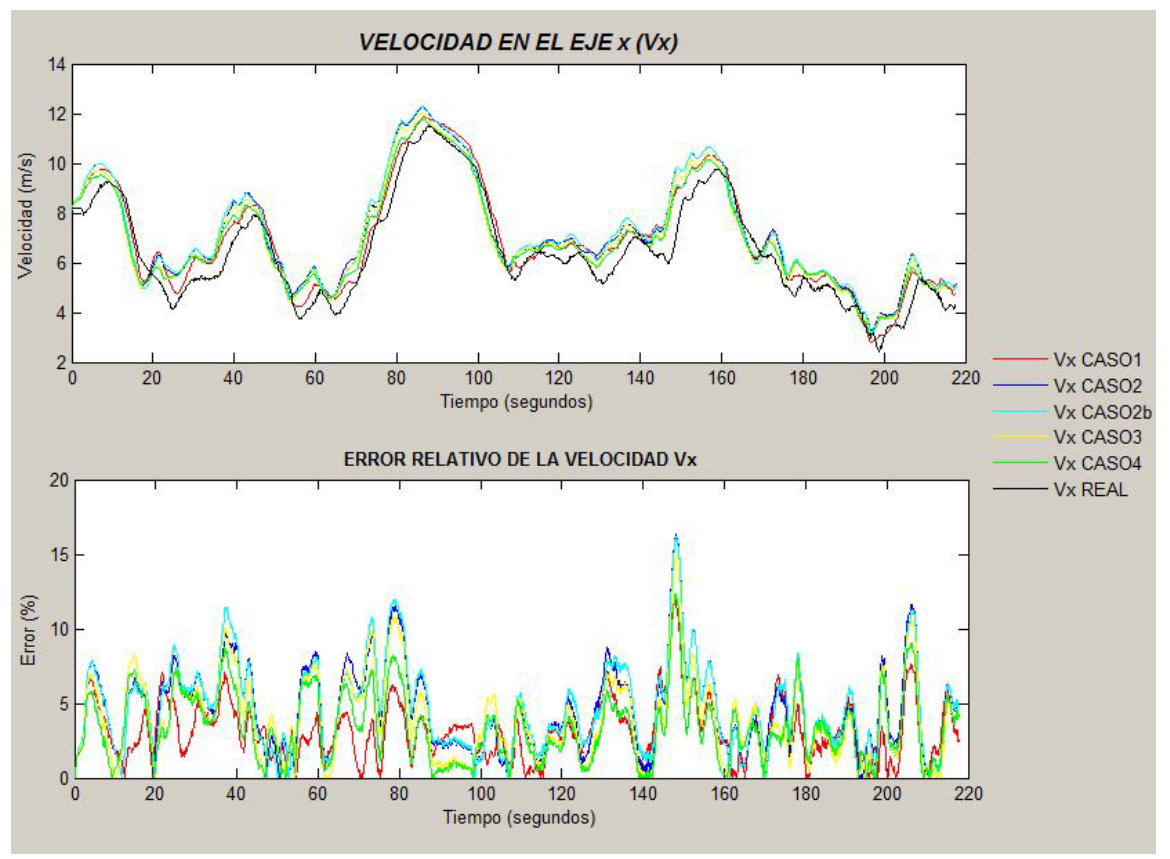

Fig. 4 - Resultados de la estimación de la velocidad longitudinal en función de la arquitectura del sistema de bajo coste propuesto.

\section{HOMENAJE}

Los autores de este artículo queremos rendir homenaje a nuestro amigo y compañero Marco Carrera Alegre, Investigador Principal de diferentes proyectos que relativos a esta materia y fallecido inesperadamente el pasado 20 de Mayo de 2015. Descanse en paz.

\section{AGRADECIMIENTOS}

La publicación de este este artículo de investigación ha sido posible gracias a la financiación proporcionada por el Ministerio de Economía y Competitividad, a través de los proyectos pertenecientes al Plan Nacional de I+D+i TRA2009-14513-C02 y TRA201238826-C02, de cuyo segundo subproyecto era Investigador Principal Marco Carrera Alegre.

La realización de los ensayos ha sido posible a la empresa LECITRAILER S.A. la cual ha proporcionado el vehículo y la cabeza tractora con la cual se ha realizado el ensayo, además de proporcionar las instalaciones para llevarlos a cabo. 


\section{REFERENCIAS}

ISO 15037-2. Road vehicles - Vehicle dynamics test methods - Part 2: General conditions for heavy vehicles and buses

ISO 11012:2009. Heavy commercial vehicles and buses -- Open-loop test methods for the quantification of on-centre handling -- Weave test and transition test

LUQUE, P. y MÁNTARAS, D. (2003) Pneumatic suspensions in semi-trailers: Part I. General considerations and simplified models. International Journal of Heavy Vehicle Systems, 10:4, 295-308.

MÁNTARAS, D.A. y LUQUE, P. (2012) Virtual test rig to improve the design and optimisation process of the vehicle steering and suspension systems. Vehicle System Dynamics, 50:10, 1563-1584.

CH ROBOTIC. Available online: www.chrobotics.com.

VBOX SPORT. Available online: http://www.vboxmotorsport.co.uk/

GENESYS. Available online: http://www.genesys-adma.de/ 